\title{
Comparison of phylobetadiversity indices based on community data from Gutianshan forest plot
}

\author{
FENG Gang ${ }^{1,2}$, ZHANG JinLong $^{1,2}$, PEI NanCai ${ }^{3}$, RAO MiDe $^{4}$, MI XiangCheng ${ }^{1 *}$, \\ REN HaiBao ${ }^{1} \&$ MA KePing ${ }^{1}$ \\ ${ }^{1}$ State Key Laboratory of Vegetation and Environmental Change, Institute of Botany, Chinese Academy of Sciences, Beijing 100093, China; \\ ${ }^{2}$ Graduate University of Chinese Academy of Sciences, Beijing 100049, China; \\ ${ }^{3}$ Research Institute of Tropical Forestry, Chinese Academy of Forestry, Guangzhou 510520, China; \\ ${ }^{4}$ College of Chemistry and Life Sciences, Zhejiang Normal University, Jinhua 321004, China
}

Received June 13, 2011; accepted September 28, 2011; published online December 8, 2011

Phylobetadiversity incorporates phylogenetic information and beta diversity, and can account for the ecological similarities between communities with a phylogenetic perspective. Although different phylobetadiversity indices reflect differences in different characteristics between communities, the results of different phylobetadiversity indices are not comparable. In this study we examined phylobetadiversity indices for a $24-\mathrm{hm}^{2}$ plot in the Gutianshan National Nature Reserve. It was found the abundanceweighted $D_{\mathrm{pw}}$ was almost identical to Rao's $D$ of Rao's quadratic entropy. PhyloSor had a similar ecological meaning and algorithm to UniFrac. Although $D_{\mathrm{nn}}$ was different in definition from UniFrac and PhyloSor, they were all strongly correlated. The effect of species abundance on phylobetadiversity was not significant when scales were relatively small, but was significant at larger scales. These contrasts likely resulted from reductions in evenness in communities as scales increased. $P_{\mathrm{ST}}$ and Rao's $H$ better reflected the distance-decay changes caused by spatial and habitat variation than other indices at larger scales, whereas AW- $D_{\mathrm{nn}}$ and $D_{\mathrm{nn}}$ better reflected these changes at small scales.

Scale, abundance, correlation, spatial pattern, distance decay

Citation: Feng G, Zhang J L, Pei N C, et al. Comparison of phylobetadiversity indices based on community data from Gutianshan forest plot. Chinese Sci Bull, 2012, 57: 623-630, doi: 10.1007/s11434-011-4869-1

Beta diversity is generally defined as the change in community composition along environmental gradients $[1,2]$. Because it directly links local diversity (alpha diversity) with regional diversity (gamma diversity), beta diversity is currently an important topic in community ecology $[1,2]$. Studies on the relationships between beta diversity and species characteristics, habitat gradients and limitations to seed dispersal can explain the mechanisms shaping patterns of beta diversity and test ecological hypotheses regarding the effects of regional diversity on local diversity $[3,4]$.

Recently, the consideration of phylogenetic relationships among the species making up a community has provided new insights into community ecology [5]. Based on species

*Corresponding author (email: mixiangcheng@ibcas.ac.cn) beta diversity, phylobetadiversity is defined as the phylogenetic distance among species or individual organisms of different communities [6]. Species beta diversity can be used to describe the dissimilarity of species composition among communities, but it is uninformative about the dissimilarity of phylogenetic relationships among communities [7,8]. For example, for 4 forest communities A, B, C and D located at different latitudes, all dissimilarity indices of species beta diversity between the forests of 1 means the species composition is completely different. However, the phylobetadiversity among these 4 communities may be dissimilar to different extents according to the relatedness of the communities.

Phylobetadiversity has been of considerable interest because of the potential new insights into community ecology 
it provides. A variety of definitions of phylobetadiversity and associated phylobetadiversity indices have been put forward. For example, $D_{\mathrm{pw}}$ is the mean pairwise phylogenetic distance of species or individuals among communities [9]; PhyloSor is defined as the proportion of branch length of shared species relative to total branch length of all species in two communities [10]; and PCD is defined as the extent to which the variance of a randomly selected trait in one community can be predicted by the value of the same trait in another community [11]. Presently, about 12 phylobetadiversity indices have been proposed. These indices can reflect dissimilarities among communities from different aspects, but the results using different indices are unlikely to be comparable.

The main ecological processes structuring the patterns of phylobetadiversity include niche-based deterministic and dispersal-based neutral models [6]. Niche-based deterministic models assume that phylobetadiveristy patterns are determined by habitat heterogeneity and interspecific tradeoffs in resource utilization, whereas dispersal-based neutral models hypothesize that phylobetadiversity patterns are shaped by spatial community dynamics, such as dispersal limitation [6]. Which of these indices can better reflect the changes in phylogenetic community structure along spatial and habitat gradients? So far, no studies have compared the characteristics of different phylobetadiversity indices.

This study aimed to compare properties of different phylobetadiversity indices using community data and environ-

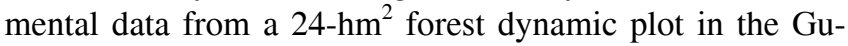
tianshan National Nature Reserve. The Gutianshan Reserve is ideal to conduct such a comparative study. Previous studies on subtropical evergreen broad-leaved forests in the Gutianshan reserve found that both niche and spatial processes have similar effects (30\% vs. $29 \%$ ) on species beta diversity [12]. Moreover, the soil nutrients and species distribution have been mapped in the reserve at high resolution.

Based on the data from the Gutianshan plot, we aimed to address 4 questions: (1) Do phylobetadiversity indices, integrated with phylogenetic information, differ from species beta diversity indices? (2) Are phylobetadiversity indices correlated with each other, and do they have similar ecological meaning? (3) Does the effect of abundanceweighting on indices of phylobetadiversity vary with scale? (4) Can these indices reflect the phylobetadiversity along spatial and habitat gradients?

\section{Materials and methods}

\subsection{Study site}

The Gutianshan subtropical evergreen broad-leaved forest dynamic plot is located in the Gutianshan National Natural Reserve in Kaihua County, Zhejiang Province, China $\left(29^{\circ} 10^{\prime} 19.4^{\prime \prime} \sim 29^{\circ} 17^{\prime} 41.4^{\prime \prime} \mathrm{N}, \quad 118^{\circ} 03^{\prime} 49.7^{\prime \prime} \sim 118^{\circ} 11^{\prime} 12.2^{\prime \prime} \mathrm{E}\right)$. The total area of the reserve is $8107 \mathrm{hm}^{2}$ and its topography is complex. The climate belongs to the middle subtropical monsoon climate zone, where the mean annual temperature is $15.3^{\circ} \mathrm{C}$, mean hottest month temperature is $28.9^{\circ} \mathrm{C}$ and mean coldest month temperature is $4.1^{\circ} \mathrm{C}$. With 140 -d precipitation, the annual precipitation is $1963.7 \mathrm{~mm}$. The area experiences, on average, 1747.5 sunshine hours and a 250-d frost-free season every year [13]. The Gutianshan forest plot is $600 \mathrm{~m}$ long and $400 \mathrm{~m}$ wide, and the altitude above sea ranges from 446.3 to $714.9 \mathrm{~m}$. The plot was established in 2005 following the Center for Tropical Forest Science census protocol [14].

\subsection{Reconstruction of community phylogeny}

We reconstructed community phylogenies following Kress et al. [15] by sequencing 3 chloroplast DNA regions ( $r b c L a$, $m a t K$ and $t r n H-p s b A$ ) of 156 woody species growing in the plot. Total DNA was extracted from leaf tissue of plant samples using the CTAB method $[16,17]$. The 3 chloroplast DNA regions were amplified and sequenced, and the nucleotide sequences were aligned using MUSCLE [18]. The three regions were assembled into a supermatrix using $\mathrm{R}$ package phylotools [19]. Three-partition GTR + GAMMA models were applied to the 3 regions separately using RAxML [20] and a community phylogeny was constructed using maximum likelihood analysis. A bootstrap analysis with 1000 replicates was conducted to assess the percentage support for each node. Finally, an ultrametric tree was obtained using the non-parametric rate smoothing approach in the r8s software package $[15,21,22]$.

\subsection{Data analysis}

Community data was obtained from the first survey of the Gutianshan $24 \mathrm{hm}^{2}$ forest plot. The survey covered 140700 woody plant individuals with diameter at breast height (dbh) $\geqslant 1 \mathrm{~cm}$ that belonged to 49 families, 104 genera and 159 species. The most abundant species were Castanopsis eyrei and Schima superba. We divided the 24-hm ${ }^{2}$ plot into 600 $20 \mathrm{~m} \times 20 \mathrm{~m}, 15040 \mathrm{~m} \times 40 \mathrm{~m}$, and $24100 \mathrm{~m} \times 100 \mathrm{~m}$ separate samples.

First, we conducted a Spearman correlation analysis of the 12 phylobetadiversity indices at the same spatial scale. Then we tested for correlations at 4 spatial scales between the 3 pairs of indices ( $D_{\mathrm{pw}}$ vs. AW- $D_{\mathrm{pw}}, D_{\mathrm{nn}}$ vs. AW- $D_{\mathrm{nn}}$, and $\prod_{\mathrm{ST}}$ vs. $\left.P_{\mathrm{ST}}\right)$ in which each pair differed by whether or not it was abundance-weighted. Finally, we ran a Partial Mantel test with the phylobetadiversity indices values and spatial distance and/or environmental distance to compare the extent that the variation of phylobetadiversity caused by spatial and environmental distance could be explained by the different indices.

The environmental variables included four topographic 
factors (mean elevation, convexity, slope, and aspect) [12] and 20 soil factors (total $\mathrm{C}$, total $\mathrm{N}$, total $\mathrm{P}$, extractable $\mathrm{Fe}$, extractable $\mathrm{Mn}$, extractable $\mathrm{Zn}$, extractable $\mathrm{Cu}$, extractable $\mathrm{K}$, extractable $\mathrm{P}$, extractable $\mathrm{Ca}$, extractable $\mathrm{Mg}$, extractable $\mathrm{Na}$, extractable $\mathrm{B}$, extractable $\mathrm{Si}$, extractable $\mathrm{Al}$, extractable $\mathrm{N}, \mathrm{pH}, \mathrm{N}$ mineralization rate, bulk density, and soil moisture). Topographic factors can reflect soil moisture and nutrients indirectly. Because species in different conditions of soil moisture and nutrition have different competitive abilities, soil moisture and nutrition can affect the distribution of plants directly, so we used topographic and soil factors to represent ecological niche processes and spatial distance to represent spatial processes. According to geostatistical methods [23], we conducted soil sampling in $30 \mathrm{~m} \times 30 \mathrm{~m}$ grids at different scales and standardized the measured factors with different methods depending on their attributes: $\mathrm{pH}$ values of every sample were standardized, the other soil factors were log-transformed, aspects of samples were transformed with $\sin$ (aspect) and cos(aspect) values to represent the extent of aspects facing south and east, and the other topographic factors were standardized with the same method as soil $\mathrm{pH}$ values.

We conducted principal component analysis (PCA) to determine the extent of variables that contributed to the principal components. The first principal component, in which mean elevation was the most important contributing variable, explained $92.9 \%$ of the information. The loading matrix of the environmental factors of the PCA is presented in Table S1. Thus we used the values for the first axis of every sample to represent habitat factors when calculating environmental distance between samples. Finally, we applied variance partitioning to phylobetadiversity values with spatial distance and/or environmental distance.

\subsection{Phylobetadiversity indices}

(i) $D_{\text {pw }} \cdot D_{\text {pw }}$ calculates the mean pairwise phylogenetic distance of different species or individuals among communities [9]. The algorithms are

$$
D_{\mathrm{PW}}=\frac{\sum_{i=1}^{n_{k_{1}}} \overline{\delta_{i k_{2}}}+\sum_{j=1}^{n_{k_{2}}} \overline{\delta_{j k_{1}}}}{n_{k_{1}}+n_{k_{2}}}
$$$$
\mathrm{AW}-D_{\mathrm{pw}}=\frac{\sum_{i=1}^{n_{k_{1}}} f_{i} \overline{\delta_{i k_{2}}}+\sum_{j=1}^{n_{k_{2}}} f_{j} \overline{\delta_{j k_{1}}}}{2} \text {, species } i \neq \text { species } j \text {, }
$$

where $\overline{\delta_{i k_{2}}}$ is the mean pairwise phylogenetic distance between species $i$ in community $k_{1}$ and all species in community $k_{2} ; n_{k_{1}}$ represents the number of species in community $k_{1} ; f_{i}$ is the relative abundance of species $i$ in community $k_{1}$ [24]; and AW- $D_{\mathrm{pw}}$ is the abundance-weighted $D_{\mathrm{pw}}$.

(ii) $D_{\mathrm{nn}} \cdot \quad D_{\mathrm{nn}}$ is defined as the mean phylogenetic distance between a species in community $A$ and its most- related species in community $B$ [9]. The formulas are

$$
\begin{aligned}
D_{\mathrm{nn}} & =\frac{\sum_{i=1}^{S_{A}} \min \delta_{i B}+\sum_{j=1}^{S_{B}} \min \delta_{j A}}{S_{A}+S_{B}}, \\
\mathrm{AW}-D_{\mathrm{nn}} & =\frac{\sum_{i=1}^{S_{A}} f_{i} \min \delta_{i B}+\sum_{j=1}^{S_{B}} f_{j} \min \delta_{j A}}{2}, \\
\text { species } i & \neq \text { species } j,
\end{aligned}
$$

where $S_{A}$ is the number of species in community $A$; $\min \delta_{j A}$ represents the phylogenetic distance between species $j$ in community $B$ and its closest relative species in community $A ; f_{i}$ is the relative abundance of species $i$ in community $A[24,25]$; and $\mathrm{AW}-D_{\mathrm{nn}}$ is the abundanceweighted $D_{\text {nn }}$.

(iii) PhyloSor. PhyloSor (Phylogenetic Sørensen index) is the proportion of branch length between shared species to total branch length of all species in two communities. As indicated in the name of this index, PhyloSor is a derivative of the Sørensen index (Sor), which is the proportion of shared species in relation to the total number of species in the communities. The larger the Sørensen value, the more similar the species composition is among communities; a larger PhyloSor value indicates closer community relationships. PhyloSor and Sørensen values are calculated by the formulas:

$$
\begin{gathered}
\operatorname{Sor}_{i j}=\frac{2 \times S_{i j}}{S_{i}+S_{j}}, \\
\text { PhyloSor }_{i j}=\frac{2 \times B L_{i j}}{B L_{i}+B L_{j}},
\end{gathered}
$$

where $S_{i j}$ is the number of species shared by two communities; $S_{i}$ is the total number of species in community $i$ [26]; $B L_{i j}$ represents branch length between species shared by two communities; and $B L_{i}$ is the branch length between all species of community $i$ [10].

(iv) UniFrac. UniFrac (unique fraction) is defined as the percentage of branch length between species unique to one community [27]. The Jaccard index [1], which is similar to UniFrac, is an index of species beta diversity. Formulas for these two indices are

$$
\begin{gathered}
\text { Jaccard }=\frac{b+c}{a+b+c}, \\
\text { UniFrac }=\frac{B+C}{A+B+C},
\end{gathered}
$$

where $a$ is number of shared species between communities; $b$ is number of species unique to community $1 ; c$ is number of species unique to community $2 ; A$ represents branch length between species shared by the two communities; $B$ represents branch length of species unique to community 1 ; and $C$ represents branch length of species unique to community 2. UniFrac and PhyloSor have similar ecological meanings; the only difference is that the former is an index of dissimilarity between communities, whereas the latter is an index of similarity. 
(v) Rao's $D$. Rao's $D$ has the same meaning as AW$D_{\text {pw. It is computed as }}$

$$
D_{k l}=\sum_{i} \sum_{j} t_{i j} x_{k i} x_{l j},
$$

where $x_{k i}$ is relative abundance of species $i$ in community $k$; and $t_{i j}$ represents phylogenetic distance between species $i$ and $j[28]$.

(vi) Rao's $H$. Rao's $H$ is an index of phylogenetic diversity without consideration of the effect of phylogenetic diversity within communities. The formula is

$$
H_{k l}=D_{k l}-\left(D_{k k}+D_{l l}\right) / 2,
$$

where $D_{k l}$ is the mean pairwise phylogenetic distance between communities; and $D_{k k}$ and $D_{l l}$ are the mean pairwise phylogenetic distances within communities $k$ and $i$, respectively.

(vii) $\prod_{\mathrm{ST}}$ and $P_{\mathrm{ST}}$. $\quad \prod_{\mathrm{ST}}$ is calculated as the mean pairwise phylogenetic distance among communities minus the mean pairwise phylogenetic distance within communities. $\prod_{\text {ST }}$ only uses $(0,1)$ data, whereas $P_{\mathrm{ST}}$ contains species abundance information [8]. The indices are calculated by

$$
\begin{gathered}
\prod_{\mathrm{ST}}=\frac{\Delta_{T}^{P}-\Delta_{S}^{P}}{\Delta_{T}^{P}}, \\
P_{\mathrm{ST}}=\frac{D_{T}^{P}-D_{S}^{P}}{D_{T}^{P}},
\end{gathered}
$$

where $\Delta_{T}^{P}$ is total phylogenetic diversity among all communities that can be understood as phylogenetic $\gamma$ diversity; $\Delta_{S}^{P}$ represents the mean value of phylogenetic diversity within communities that can be understood as phylogenetic alpha diversity; and $D_{T}^{P}$ and $D_{S}^{P}$ are abundance-weighted phylogenetic gamma and alpha diversity, respectively.

(viii) PCD. PCD (phylogenetic community dissimilarity) can be broken up into a non-phylogenetic component $\left(\mathrm{PCD}_{\mathrm{c}}\right)$, which reflects shared species among communities, and a phylogenetic component $\left(\mathrm{PCD}_{\mathrm{p}}\right)$, which reflects relatedness of different species among communities. Interpretation of this phylogenetic beta diversity required the assumption that the evolutionary process of a non-selected trait is random, which is Brownian motion. Given the phylogenetic relationship between two communities, PCD represents the extent that variance of the trait in community 1 can be predicted by the same trait value in community 2 :

$$
\mathrm{PCD}=\frac{n_{1} \mathrm{PSV}_{1 \mid 2}+n_{2} \mathrm{PSV}_{2 \mid 1}}{n_{1} \mathrm{PSV}_{1}+n_{2} \mathrm{PSV}_{2}} \times \frac{1}{\bar{D}\left(n_{1} \times n_{2} \times C_{\text {pool }}\right)} \text {, }
$$

where $n_{1}$ means species number in community 1 ; $\operatorname{PSV}_{1 \mid 2}$ represents the variance of the trait among species in community 1 given the trait variance in community 2 [29]; $\mathrm{PSV}_{1}$ is the variance of the trait among species in community 1 ; and $\bar{D}\left(n_{1} \times n_{2} \times C_{\text {pool }}\right)$ is used to remove the deviation caused by $n_{1}, n_{2}[11]$.

\section{Results}

\subsection{Correlations between indices}

We investigated correlations between phylobetadiversity indices caused by the integration of phylogenetic information with species beta diversity using Spearman correlation analysis between UniFrac and PhyloSor, UniFrac and Jaccard [1], and PhyloSor and Sørensen [26]. These three pairs of indices have similar ecological meaning. Results at different spatial scales are shown in Table 1. Because Sørensen and Jaccard are the species beta diversity of PhyloSor and UniFrac, correlations between the phylobetadiversity indices change after integration of phylogenetic information (Table 1).

We calculated 12 phylobetadiversity indices (if weighted and unweighted abundances are included), among which five pairs were highly correlated (Figure 1, Tables 2 and 3). The highly correlated pairs were AW- $D_{\mathrm{pw}}$ and Rao's $D$, PhyloSor and UniFrac, $D_{\mathrm{nn}}$ and PhyloSor, $D_{\mathrm{nn}}$ and UniFrac, and Rao's $H$ and $P_{\mathrm{ST}}$.

Since the indices PCD and PCDp account for the covariance of phylogenetic distance between species, we compared them with UniFrac and $\prod_{\mathrm{ST}}$, which also take the covariance of phylogenetic distance between species into consideration (Table 3 for the spatial scale of $100 \mathrm{~m} \times 100 \mathrm{~m}$, and see Tables S2 and S3 for all other spatial scales).

\subsection{Effect of abundance-weighting on phylobetadiver- sity}

We conducted Spearman correlation analysis on three pairs of indices that differed in being either abundance-weighted or not at 4 spatial scales, namely $10 \mathrm{~m} \times 10 \mathrm{~m}, 20 \mathrm{~m} \times 20 \mathrm{~m}$, $40 \mathrm{~m} \times 40 \mathrm{~m}$, and $100 \mathrm{~m} \times 100 \mathrm{~m}$. The difference between each pair of indices was relatively small and became larger with increased spatial scale (Figure 2). This could be explained by the change of species evenness with increased scale. Sampling of species abundance distribution at different scales supported this hypothesis (Figure 3).

Table 1 Spearman rank correlation coefficients between UniFrac and PhyloSor, UniFrac and Jaccard and PhyloSor and Sørensen at three spatial scales

\begin{tabular}{cccc}
\hline Indices & $20 \mathrm{~m} \times 20 \mathrm{~m}$ & $40 \mathrm{~m} \times 40 \mathrm{~m}$ & $100 \mathrm{~m} \times 100 \mathrm{~m}$ \\
\hline UniFrac and PhyloSor & -1 & -1 & -1 \\
UniFrac and Jaccard & 0.81 & 0.82 & 0.81 \\
PhyloSor and Sørensen & 0.81 & 0.82 & 0.81 \\
\hline
\end{tabular}



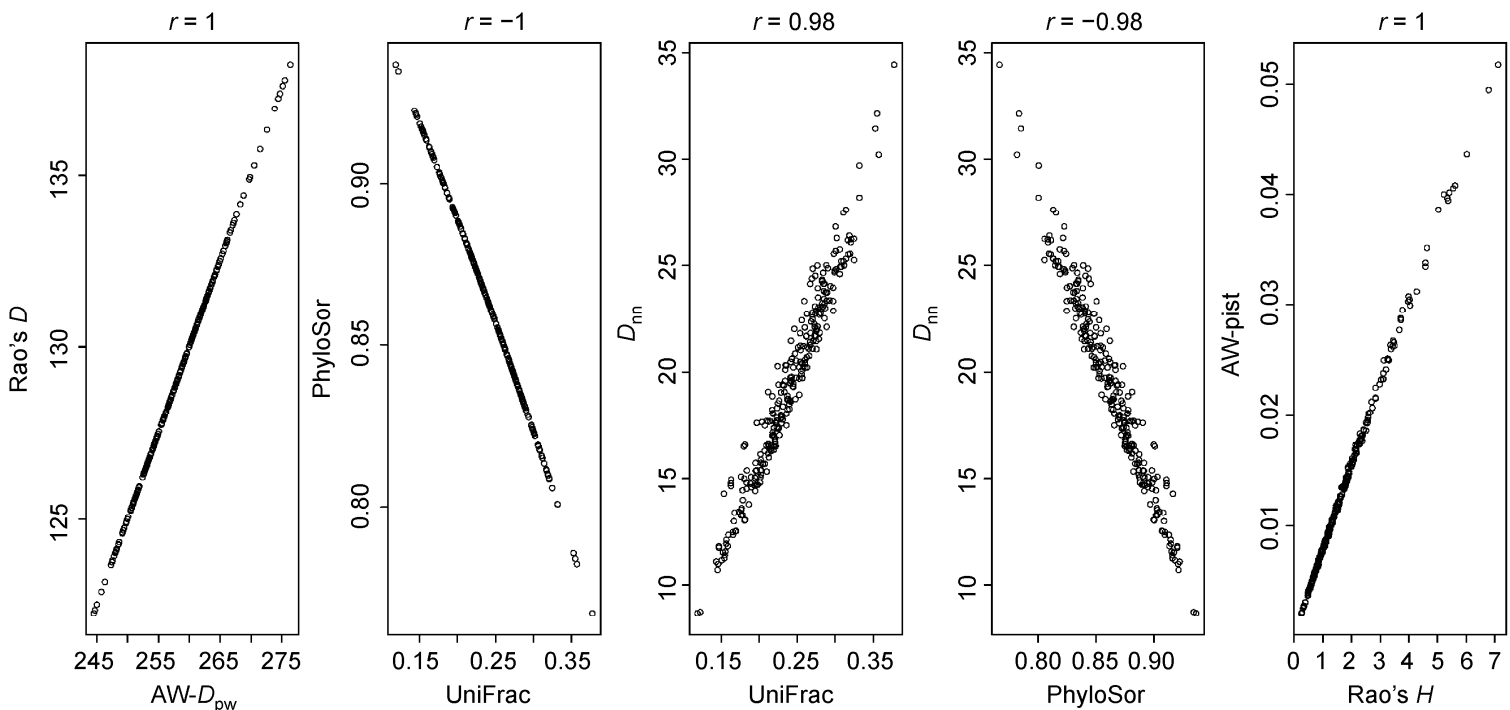

Figure 1 Spearman rank correlation coefficients between phylobetadiversity indices at a spatial scale of $100 \mathrm{~m} \times 100 \mathrm{~m}$.

Table 2 Spearman rank correlation coefficients between phylobetadiversity indices at a spatial scale of $100 \mathrm{~m} \times 100 \mathrm{~m}$; high correlation coefficients were bolded.

\begin{tabular}{|c|c|c|c|c|c|c|c|c|c|}
\hline 0.26 & 0.47 & 0.34 & 0.31 & -0.32 & 0.32 & 1 & 0.47 & 0.41 & $P_{\mathrm{ST}}$ \\
\hline 0.04 & 0.36 & 0.6 & 0.6 & -0.55 & 0.55 & 0.42 & 0.36 & $\prod_{\mathrm{ST}}$ & \\
\hline-0.05 & 1 & 0.11 & 0.17 & -0.11 & 0.11 & 0.49 & Rao's $D$ & & \\
\hline 0.25 & 0.49 & 0.34 & 0.32 & -0.32 & 0.32 & Rao's $H$ & & & \\
\hline-0.08 & 0.11 & 0.98 & 0.66 & -1 & UniFrac & & & & \\
\hline-0.02 & 0.17 & 0.66 & $\mathrm{AW}-D_{\mathrm{nn}}$ & & & & & & \\
\hline-0.01 & 0.11 & $D_{\mathrm{nn}}$ & & & & & & & \\
\hline-0.05 & $\mathrm{AW}-D_{\mathrm{pw}}$ & & & & & & & & \\
\hline$D_{\mathrm{pw}}$ & & & & & & & & & \\
\hline
\end{tabular}

Table 3 Spearman rank correlation coefficients between phylobetadiversity indices at a spatial scale of $100 \mathrm{~m} \times 100 \mathrm{~m}$

\begin{tabular}{|c|c|c|c|}
\hline 0.27 & 0.57 & 0.24 & $\prod_{\mathrm{sT}}$ \\
\hline 0.86 & 0.43 & UniFrac & \\
\hline 0.53 & $\mathrm{PCD}_{\mathrm{p}}$ & & \\
\hline PCD & & & \\
\hline
\end{tabular}

\subsection{Ability of indices to reflect variation in community composition along habitat and spatial gradients}

To find which index can best reflect the variation of phylobetadiversity along habitat and spatial gradients, we used a Partial Mantel test to partition phylobetadiversity with respect to habitat and/or spatial gradients (Table 4 for $100 \mathrm{~m}$ $\times 100 \mathrm{~m}$ spatial scale, and see Tables S4 and S5 for all other scales).

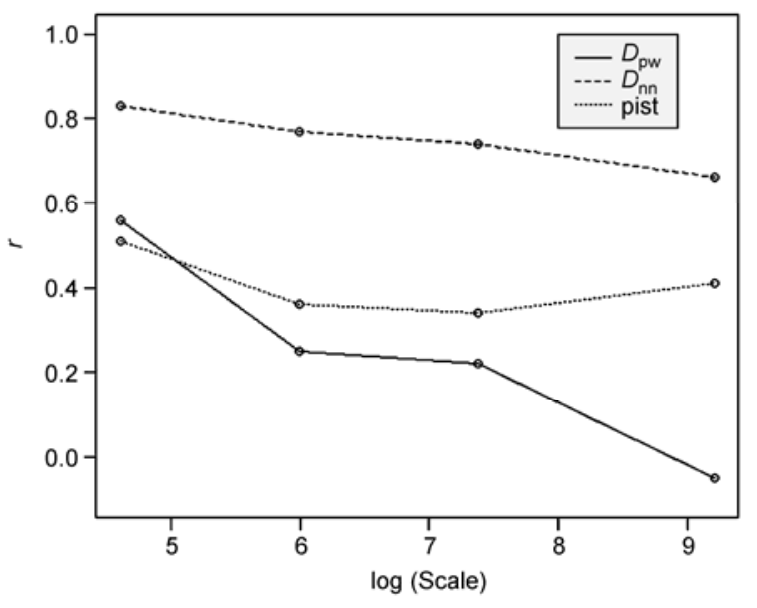

Figure 2 Effect of spatial scale on correlation coefficients between abundance-weighted and non-weighted phylobetadiversity indices. 


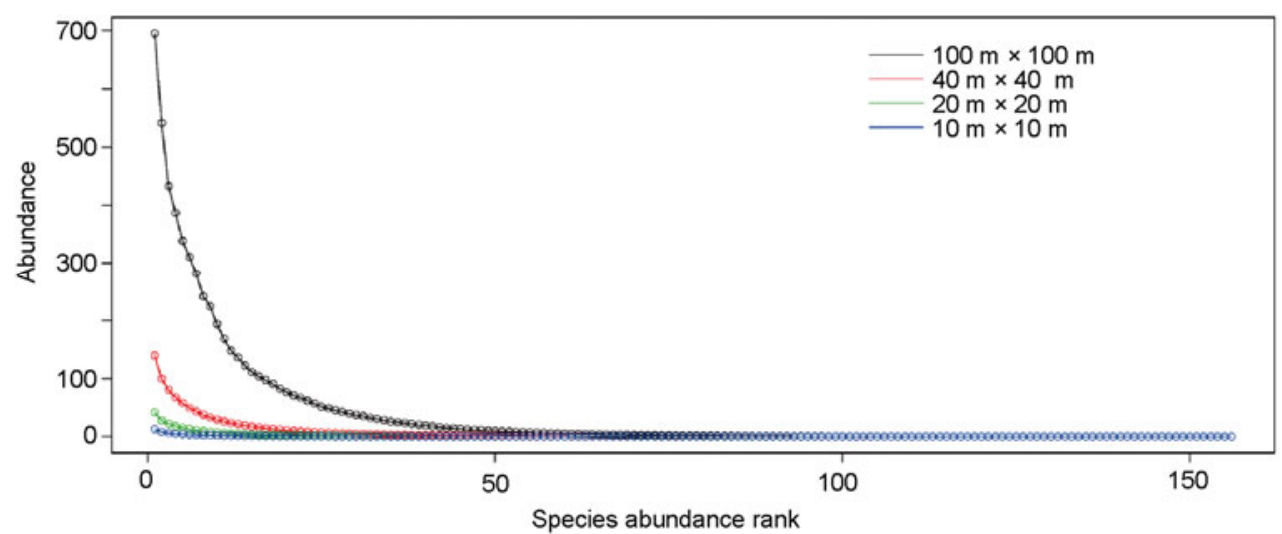

Figure 3 Absolute abundances of abundance rankings at different spatial scales.

Table 4 Results of a variation partitioning between phylobetadiversity values and habitat and spatial gradients at the spatial scale of $100 \mathrm{~m} \times 100 \mathrm{~m}$

\begin{tabular}{lccccc}
\hline \multicolumn{1}{c}{ Index } & $a+b+c$ & $a+b$ & $b+c$ & $a$ & $c$ \\
\hline$D_{\mathrm{pw}}$ & 0.025 & -0.004 & 0.022 & 0.003 & 0.029 \\
AW- $D_{\mathrm{pw}}$ & 0.065 & 0.002 & 0.067 & -0.002 & 0.063 \\
$D_{\mathrm{nn}}$ & 0.225 & 0.097 & 0.206 & 0.018 & 0.128 \\
AW- $D_{\mathrm{nn}}$ & 0.303 & 0.106 & 0.29 & 0.013 & 0.197 \\
UniFrac & 0.233 & 0.091 & 0.219 & 0.014 & 0.142 \\
PhyloSor & 0.236 & 0.091 & 0.222 & 0.014 & 0.145 \\
Rao's $D$ & 0.065 & 0.002 & 0.067 & -0.002 & 0.063 \\
Rao's $H$ & 0.321 & 0.053 & 0.323 & -0.002 & 0.268 \\
$\prod_{\text {sT }}$ & 0.216 & 0.121 & 0.18 & 0.037 & 0.096 \\
$P_{\text {ST }}$ & 0.328 & 0.056 & 0.33 & -0.002 & 0.272 \\
PCD & 0.018 & 0.021 & 0.005 & 0.013 & -0.002 \\
PCD & 0.002 & 0.001 & -0.003 & 0.005 & 0 \\
\hline
\end{tabular}

\section{Discussion}

Based on species beta diversity, phylobetadiversity can provide new insights into species coexistence from phylogenetic relationships among species [6]. Niche conservation during evolutionary history plays a critical role in determining species distribution and offers a basis for phylobetadiversity studies [30,31]. Phylobetadiversity can be used to explore mechanisms of biodiversity maintenance and may have a higher utility than species beta diversity as a conservation criterion for management decisions.

\subsection{Correlations between indices}

Correlation analysis of phylobetadiversity indices can help us to understand better the ecological meaning of phylobetadiversity and avoid confusion. Both $\mathrm{AW}-D_{\mathrm{pw}}$ and Rao's $D$ represent abundance-weighted mean pairwise phylogenetic distance and results of the two indices are identical, so we can choose one of these indices in future studies; PhyloSor and UniFrac are derived from the Sørensen and
Jaccard indices and have similar ecological meanings, i.e., the proportion of branch length shared between species to total branch length of all species in two communities. The only difference between the two indices is that PhyloSor is an index of similarity, whereas UniFrac is an index of dissimilarity. Rao's $H$ and $P_{\mathrm{ST}}$ also have similar ecological meanings and are highly correlated; what needs further study is the finding that correlations between $D_{\mathrm{nn}}$ and PhyloSor, $D_{\mathrm{nn}}$ and UniFrac are also highly correlated. $D_{\mathrm{nn}}$ represents the mean nearest phylogenetic distance between two communities, whereas PhyloSor and UniFrac are the proportion of branch length between species shared relative to total branch length of all species in two communities. All of these indices represent the difference between the terminals of phylogenetic trees of species from two different communities, which may explain their strong correlation.

\subsection{Effect of abundance-weighting on indices}

Abundance, which reflects differences in species evenness between communities, is an important concept in community ecology. Lozupone et al. [32] found that abundanceweighted indices are suitable in studies that investigate changes in species abundance when the mechanisms may be correlated with subtle environmental changes. On the other hand, abundance-unweighted indices are used mainly to discuss factors limiting species presence.

The correlation analysis of the three pairs of indices at four spatial scales showed a decrease in strength of correlation with increasing spatial scales. In other words, when the scale is small, effect of abundance is not significant and the effect becomes more obvious as scale increases. Thus if the scale applied in an investigation is relatively small, both of each of the pairs might not need to be calculated.

\subsection{Performance of indices that reflect habitat and spatial gradients}

It has been shown widely that similarities between commu- 
nities decrease as spatial distances between the communities increase [33]. There are two main mechanisms that explain this phenomenon: (1) habitat characteristics change with increased separation distance, and affect community composition and can be interpreted by the niche hypothesis [34]; and (2) according to neutral theory [35], although the habitat between communities may be similar, similarity between communities will decrease with increased spatial distance because of the limiting dispersal abilities of organisms.

Phylobetadiversity, as the measurement of phylogenetic distance between communities, should also decay with distance. Results of variance partitioning of phylobetadiversity between habitat and spatial distance indicate that $P_{\mathrm{ST}}$ and Rao's $H$ can best reflect distance decay along habitat and spatial gradients in subtropical forest communities at the spatial scale of $100 \mathrm{~m} \times 100 \mathrm{~m}$. At this scale, habitat in conjunction with spatial distance explained $32.77 \%$ and $32.1 \%$ of the variance of Rao's $H$ and $P_{\mathrm{ST}}$, respectively; however, as the spatial scale decreased to $20 \mathrm{~m} \times 20 \mathrm{~m}$ and $40 \mathrm{~m} \times 40 \mathrm{~m}$, distance decay along spatial and habitat gradients was best reflected by AW- $D_{\mathrm{nn}}$ and $D_{\mathrm{nn}}$. At a scale of $20 \mathrm{~m} \times 20 \mathrm{~m}$ the variance of AW- $D_{\mathrm{nn}}$ and $D_{\mathrm{nn}}$ explained by habitat factors and spatial distance was $13.4 \%$ and $9.375 \%$, respectively, and at the $40 \mathrm{~m} \times 40 \mathrm{~m}$ scale $18.63 \%$ and $13.47 \%$ were explained by the same variables, respectively. Therefore we recommend using $P_{\mathrm{ST}}$ and Rao's $\mathrm{H}$ when the scales are relatively large, and $\mathrm{AW}-D_{\mathrm{nn}}$ and $D_{\mathrm{nn}}$ when scales are smaller, for studies on effects of different factors on phylobetadiversity.

We found that $\mathrm{AW}-D_{\mathrm{pw}}$ and Rao's $D$, PhyloSor and UniFrac, $D_{\mathrm{nn}}$ and PhyloSor, $D_{\mathrm{nn}}$ and UniFrac, Rao's $H$ and $P_{\mathrm{ST}}$ are strongly correlated. $D_{\mathrm{pw}}$, AW- $D_{\mathrm{pw}}$, Rao's $D$, Rao's $H, P_{\mathrm{ST}}$ and $\prod_{\mathrm{ST}}$ are based on the mean pairwise phylogenetic distance, which reflects branch differences close to the phylogenetic tree root between communities and can more effectively reflect the habitat difference than other indices. Calculations of $D_{\mathrm{nn}}, \mathrm{AW}-D_{\mathrm{nn}}$, UniFrac and PhyloSor are measures of differences in phylogenetic tree terminals, which are differences in evolutionary distinctiveness [36] and may be used to reflect differences in resource utilization strategies. For these indices, abundance-weighting at small scales has little effect, but the effect of abundance increases at larger scales. At large spatial scales, habitat and space explain $P_{\mathrm{ST}}$ and Rao's $\mathrm{H}$ best, but at smaller spatial scales AW- $D_{\mathrm{nn}}$ and $D_{\mathrm{nn}}$ are better explained by habitat and space.

Phylobetadiversity provides a new perspective on relationships between communities; therefore, studies combining species beta diversity and phylobetadiversity may be more comprehensive. This study analyzed correlations among phylobetadiversity indices, effects of abundanceweighting on phylobetadiversity indices at different spatial scales, and the potential of the indices to reflect spatial and habitat gradients. The findings might be helpful to researchers interested in phylogenetic ecology.
We thank Dr. Nathan Swenson for his insightful suggestions on this manuscript, Liu Xiaojuan, Man Xingxing and Song Kai for their help with data processing. Dr. Liwen Zhang supplied the soil data and a number of teachers and workmates also assisted in the Gutianshan 24 ha plot. We also thank Christine Verhille, University of British Columbia, for her assistance with English language and grammatical editing of the manuscript. This work was supported by Key Innovation Project of Chinese Academy of Sciences (KZCX2-EW-Z-5) and National Natural Science Foundation of China (31170401).

1 Whittaker R H. Vegetation of the Siskiyou Mountains, Oregon and California. Ecol Monogr, 1960, 30: 280-338

2 Cody M L. Towards a theory of continental species diversities: bird distributions over Mediterranean habitat gradients. In: Cody M L, Diamond J M, eds. Ecol Evolcomm. Harvard: Harvard University Press, 1975, 214-257

3 Koleff P, Gaston K J, Lennon J J. Measuring beta diversity for presence-absence data. J Anim Ecol, 2003, 72: 367-382

4 Chen S B , Ouyang Z Y, Xu W H, et al. The research progress of beta diversity (in Chinese). Biodiver Sci, 2010, 18: 323-335

5 Webb C O. Exploring the phylogenetic structure of ecological communities: An example for rain forest trees. Am Nat, 2000, 156: 145-155

6 Graham C H, Fine P V A. Phylogenetic beta diversity: Linking ecological and evolutionary processes across space in time. Ecol Lett, 2008, 11: 1265-1277

7 Chave J, Chust G, Thébaud C. The importance of phylogenetic structure in biodiversity studies. In: Storch D, Marquet, Brown J H, eds. Scaling Biodiversity, Institute Editions, Santa Fe, 2007. 151-167

8 Hardy O J, Senterre B. Characterizing the phylogenetic structure of communities by an additive partitioning of phylogenetic diversity. J Anim Ecol, 2007, 95: 493-506

9 Webb C O, Ackerly D D, Kembel S W. Phylocom: Software for the analysis of phylogenetic community structure and trait evolution. Bioinformatics, 2008, 24: 2098-2100

10 Bryant J A, Lamanna C, Morlon H, et al. Microbes on mountainsides: Contrasting elevational patterns of bacterial and plant diversity. Proc Natl Acad Sci USA, 2008, 105: 11505-11511

11 Ives A R, Helmus M R. Phylogenetic metrics of community similarity. Am Nat, 2010, 176: E128-E142

12 Pierre L, Mi X C, Ren H B, et al. Partitioning beta diversity in a subtropical broad-leaved forest of China. Ecology, 2009, 90: 663-674

13 Lou L H, Jin S H. Spermatophyta flora of Gutianshan Nature Reserve in Zhejiang (in Chinese). J Beijing Forest Univ, 2000, 22: 33-39

14 Chen B, Mi X C, Fang T, et al. Gutianshan Forest Dynamic Plot: Tree Species And Their Distribution Patterns (in Chinese). Beijing: China Forestry Publishing House, 2009

15 Kress W J, Erickson D L, Jones F A, et al. Plant DNA barcodes and a community phylogeny of a tropical forest dynamics plot in Panama. Proc Natl Acad Sci USA, 2009, 106: 18621-18626

16 Doyle J J, Doyle J L. A rapid DNA isolation procedure for small quantities of fresh leaf tissue. Phytochem Bull, 1987, 19: 11-15

17 Khanuja S P S, Shasany A K, Darokar M P, et al. Rapid isolation of DNA from dry and fresh samples of plants producing large amounts of secondary metabolites and essential oils. Plant Mol Biol Rep, 1999, 17: $1-7$

18 Edgar R C. MUSCLE: Multiple seq uence alignment with high accuracy and high throughput. Nucleic Acids Res, 2004, 32: 1792-1797

19 Zhang J L, Mi X C, Pei N C. Phylotools: Phylogenetic tools for ecologists. R package version 0.0.7.4. 201019

20 Alexandros S. RAxML-VI-HPC: Maximum likelihood-based phylogenetic analyses with thousands of taxa and mixed models. Bioinformatics, 2006, 22: 2688-2690

21 Sanderson MJ. r8s: Inferring absolute rates of molecular evolution and divergence times in the absence of a molecular clock. Bioinformatics, 2003, 19: 301-302

22 Lemey P, Rambaut A, Drummond A J, et al. Bayesian Phylogeography finds its roots. PLoS Comput Biol, 2009, 9: e1000520

23 Zhang L W, Mi X C, Shao H B, et al. Strong plant-soil associations 
in a heterogeneous subtropical broad-leaved forest. Plant Soil, 2011, doi: 10.1007/s11104-001-0839-2

24 Swenson N G, Anglada-Cordero P, Barone J A. Deterministic tropical tree community turnover: Evidence from patterns of functional beta diversity along an elevational gradient. Proc R Soc London Ser B, 2011, 278: 877-884

25 Ricotta C, Burrascano S. Testing for differences in beta diversity with asymmetric dissimilarities. Ecol Indic, 2009, 9: 719-724

26 Krebs C J. Ecological Methodology. Menlo Park, CA: AddisonWesley, 1999

27 Lozupone C, Hamady M, Knight R. UniFrac-An online tool for comparing microbial community diversity in a phylogenetic context. BMC Bioinformatics, 2006, 7: 371-384

28 Rao C R. Diversity and dissimilarity coefficients: A unified approach. Theor Popul Biol, 1982, 21: 2443

29 Helmus M R, Bland T J, Williams C K, et al. Phylogenetic measures of biodiversity. Am Nat, 2007, 169: E68-E83
30 Donoghue M J. A phylogenetic perspective on the distribution of plant diversity. Proc Natl Acad Sci USA, 2008, 105: 11549-11555

31 Cavender-Bares J, Ackerly D D, Baum D A, et al. Phylogenetic overdispersion in Floridian oak communities. Am Nat, 2004, 163: 823-843

32 Lozupone C A, Hamady M, Kelley S T, et al. Quantitative and qualitative beta diversity measures lead to different insights into factors that structure microbial communities. Appl Environ Microbiol, 2007, 73: $1576-1585$

33 Soininen J, McDonald R, Hillebrand H. The distance decay of similarity in ecological communities. Ecography, 2007, 30: 3-12

34 Nekola J C, White P S. The distance decay of similarity in biogeography and ecology. J Biogeogr, 1999, 26: 867-878

35 Hubbell S P. The unified neutral theory of biodiversity and biogeography. Princeton: Princeton University Press, 2001

36 Redding D W, Mooers A O. Incorporating evolutionary measures into conservation prioitization. Conserv Biol, 2006, 20: 1670-1678

Open Access This article is distributed under the terms of the Creative Commons Attribution License which permits any use, distribution, and reproduction in any medium, provided the original author(s) and source are credited.

\section{Supporting Information}

Table S1 Principal component analysis of environmental factors at the spatial scale of $100 \mathrm{~m} \times 100 \mathrm{~m}$

Table S2 Spearman rank correlation coefficients between phylobetadiversity indices at spatial scale of $40 \mathrm{~m} \times 40 \mathrm{~m}$

Table S3 Spearman rank correlation coefficients between phylobetadiversity indices at spatial scale of $20 \mathrm{~m} \times 20 \mathrm{~m}$

Table S4 Results of Mantel test between phylobetadiversity values and habitat and spatial gradients at a spatial scale of $40 \mathrm{~m} \times 40 \mathrm{~m}$

Table S5 Results of Mantel test between phylobetadiversity values and habitat and spatial gradients at a spatial scale of $20 \mathrm{~m} \times 20 \mathrm{~m}$

The supporting information is available online at csb.scichina.com and www.springerlink.com. The supporting materials are published as submitted, without typesetting or editing. The responsibility for scientific accuracy and content remains entirely with the authors. 